\title{
A Research and Reflection on Yang Deyu's Translation Career and His Practices in Poetry Translation
}

\begin{abstract}
Jiana Liu
Department of Foreign Language, Guangzhou Xinhua University, Guangzhou, Guangdong Province, China.

hopeful0606@163.com

Abstract

Yang Deyu, the renowned poetry translator, divided his practices in poetry translation into three stages. In the first stage, he translated English metrical poetry into "semi-free vernacular poetry"; in the second stage, his translation was in the process of transition from the style of "semi-free" to the style of metrical; in the third stage, translating English metrical poetry into Chinese metrical poetry became his translation method. After learning Yang Deyu's life story and analyzing his representative poems of every stage, this paper found that Yang's translation career and his exploration of English poetry translation are profoundly influenced by his life experience. In his exploration, great progress has been made in preserving the metrical characteristics of English metrical poetry, however, "dun for foot" is still questionable in revealing the rhythmic foot pattern of English poetry and the rhythmic sound pattern in English poetry remains untouched in his translation. To maintain the poeticity and poetic form in English poetry, a better way, "dou" for foot and ping-ze for iambic will be employed in translation.
\end{abstract}

Key words: Yang Deyu; “dun” for foot; “dou” for foot; ping-ze for iambic

\section{对杨德豫的翻译生涯与其译诗实践的探索与反思}

\author{
刘家妠
}

广州新华学院, 外国语学院, 广州, 广东省, 中国

hopeful0606@163.com

\begin{abstract}
摘要
译诗名家杨德豫将自己的译诗实践分为三个阶段。第一阶段，他将英语格律诗译成 “半自由体” 的白话诗; 第 二阶段，其译诗从半自由体向格律体过渡；第三阶段，其译诗走向以格律体译格律体。在对杨德豫的生平和他 各个阶段的译诗代表作进行研究后, 笔者发现, 杨德豫的人生经历对其译诗生涯和译诗实践的探索过程影响极 大，而随着他探索过程的推进，其三个不同阶段的译诗在保留原诗的格律特征方面也产生了变化和进步，但是 其成熟时期的译诗观—— “以顿代步” 在展现原诗的步律特征方面仍有缺憾, 而原诗的音律特征在其翻译中也 一直被忽视。笔者将对此进行反思，并应用更能展现原诗诗形和诗意的翻译方法—— “以逗代步” “ “以平入 对抑扬”对英诗汉译进行分析。
\end{abstract}

关键词: 杨德瞀; 以顿代步; 以逗代步; 以平人对抑扬

\section{1. 引言}

杨德豫（1928 年 12 月 12 日—-2013 年 1 月 23 日）生于北平, 原籍湖南省长沙市。别名江声, 曾用 笔名张四、萧涉源、新潮、旧潮, 是我国著名的英诗 翻译家。杨德豫先生投身中国翻译事业近六十载, 曾
主持被称为“汉译诗歌第一丛书”的《诗苑译林》, 翻译 经典英诗作品无数, 所译诗集获得首届鲁迅文学奖, 全国优秀文学翻译彩虹奖。市之琳称杨译的《拜伦诗 选》“标志着我国译诗艺术的成熟”[1]；江枫称赞他的 《华兹华斯、柯尔律治诗选》为中译外文格律诗典范 
${ }^{[2]}$; 屠岸称其为“译诗天才”[3]; 北塔在《神形兼备信言 美》中赞其“驾驭语言能力令人叹服”[4]。

身为译诗名家, 杨德豫先生却鲜有专门针对译诗 的理论进行阐述，其译诗理论可见其文章、译诗诗集 的后记和前言, 以及书信和采访。在《用什么形式翻 译英语格律诗》、《华兹华斯、柯尔律治诗选后记》、

《拜伦抒情诗七十首》译后琐记、《贞女劫》译者赘 言、《朗费罗诗选》译序、《杨德豫译诗集》后记、《杨 德豫译诗集》校勘记等前言和后记中, 杨德豫对自己 的译诗理论和译诗原则略有提及。以上作品均被收入 《追寻缪斯的踪影一谈英诗汉译及其他》这一文集、 书信集中。在文集上篇“谈英诗汉译”的《译诗实践三 阶段——贞女劫>译者謷言》这一章节中, 杨德豫回 忆了自己在译诗领域二十余年的探索, 并将自己的译 诗实践分成了三个阶段 ${ }^{[5]}$ 。

\section{2. 杨德豫的生平与其译诗实践的探索过程}

杨德豫选择英诗翻译作为毕生追求是源于他对 英语的兴趣和对诗歌喜爱。对于英文, 他一直保持着 自学的习惯, 通过背英文书中的文章来学英语。他大 学期间的学习和生活也使杨德豫的英文水平得到了 显著的提升, 他大量阅读诸如《傲慢与偏见》之类的 经典的英文小说、必读的英文课本和英文课外书, 并 用英文写摘要、笔记、学习心得等。据杨德豫的女儿 杨小显回忆, 即使到了晚年, 他也依然保持着背英文 单词的习惯 $\left[{ }^{[6]}\right.$ 。杨德豫对诗歌的热爱源于他的父亲, 国学大师杨树达的引导。年幼的杨德豫熟读《诗歌易 读》、《唐诗易读》、《唐诗三百首》等, 他喜欢国文和 古典诗词, 也热爱五四以来的白话文作品, 但是这些 都不及五四的新诗对他的影响, 因为尤其喜爱读白话 新诗, 他自己也学着写一些诗歌。1 1941 年至 1942 年 之间, 他曾在沅陵《中报》上发表过一些新诗、散文 和旧诗。或许是因为杨德豫在英诗翻译方面的造诣太 高, 比较而言就使得他对自己的原创诗歌不屑一顾, 每每提及自己曾经创作的诗歌, 他都表示自己写不出 像样的旧体诗, 而这些早年写的白话诗是“毫无价值 的劣等货色" "[7]。但是, 屠岸对他的评价却极高, 说他 虽然不写诗，但是“有诗人的气质”。

杨德豫的生平不仅对他成为一名英诗翻译家产 生了影响, 其译诗实践的探索过程也和他的人生经历 有着极大的关系。在《用什么形式翻译英语格律诗》 一文中, 他总结了五四以后七十年来英语格律诗汉译 中的五大流派: 译成散文派; 译成自由诗派; 译成半 自由体派; 译成中国古典格律诗体派; 译成现代汉语 格律诗体即白话格律诗体派。在以上五大流派中, 杨 德豫应属于最后一派, 但在确立自己的翻译派别之前, 杨德豫有过“反叛”和探索, 也有过坚守和抗争, 而这 以上种种都是他的人生经历在他译诗实践过程中的 体现。

杨德豫译诗的第一阶段以 1959 年出版的《朗费 罗诗选》为代表, 特点是“把英语格律诗译成了“半自
由体”的汉语白话诗”。杨德豫将“半自由体”的汉语白 话诗定义为各行顿数既不整齐也无规律的汉语白话 诗。当时的国内译诗界, 闻一多、市之琳等人已经初 步形成了自己的译诗主张, 即把英语格律诗译成汉语 格律诗, 但是并没有受到译诗界的认可, 而“把格律 体诗译成半自由体”相习成风”, 在这种情况下, 杨德 豫声称自己不具备 “反潮流”的觉悟和勇气, 于是将 1959 年出版的《朗费罗诗选》译成了各行顿数既不整 齐也无规律的汉语白话诗。

然而，在表面的“随波逐流”之下，杨德豫内心却 藏匿着一颗对翻译精益求精的赤子之心。虽然他跟随 着当时的主流翻译方法, 使得诗选中大多数篇章顿数 既不整齐也不规律，但是也有意而为地让其中四首诗 (《人生颂》、《少女》、《烈士纪念日》和《得失》) 的 译文顿数整齐有规则。这可以看作杨德豫对当时主流 翻译观的“反叛”, 也可看作是他对英诗汉译的一次探 索性的尝试。杨德豫的“反叛”和探索的精神从他的生 平回忆中可见一斑。在生前的一次访谈中, 杨德豫直 言自己“有点犯上作乱的思想, 有些反骨”。无论是童 年幼时, 还是求学期间、军队期间, 直到晚年, 他都 保持着敢于挑战权威的“反叛”精神。但是, 也正是这 种“反叛”和特立独行的精神使得他不流于平庸, 在诗 歌翻译的领域不断地摸索和试验, 在“潮流”中坚守着 内心的一寸净土。在用业余时间翻译《朗费罗诗选》 期间, 他仍然在军队担任编辑, 所以他的这个行为, 在当时不仅不被人理解，反而被认为是不务正业，但 是杨德豫依然坚定地遵从自了己的内心，坚持用业余 的时间进行英诗翻译。

坚持不解的精神不仅贯穿了杨德豫的一生，也体 现在他对英诗汉译的探索过程中。之后杨德豫被罚劳 改, 1962 年的时候被分配到了农场宣传部去编小报, 之后又去农场教书。在农场期间, 杨德豫给人民文学 出版社写信, 并受邀翻译《鲁克丽丝受辱记》。这些年 来, 面对着劳动多、环境差等苦难, 杨德豫不仅撑了 下来, 在艰苦的劳作之中依然不改献身诗歌翻译的初 衰。据白景高、肖克勤、陈守凡等人的回忆，在农场 教书期间, 他们经常能够看到杨坐在蚊帐里满头大汗 地读书; 把自己关在六七平方米的宿舍里搞翻译; 静 静地看着自费订阅的《光明报》。杨德豫译诗的第二 阶段正是以 1962 年到 1963 年之间翻译的《鲁克丽丝 受辱记》为代表, 标志着杨德豫的译诗 “从半自由体向 格律体过渡”。

1978 年, 杨德豫的历史误会被洗清, 终于正式回 归到编辑和翻译的生涯并帮助成立了译文编辑室。在 译文编辑室成立初期, 他全国各地几经辗转, 去拜会 翻译名家, 进行组稿, 由此结识了市之琳、罗念生、 绿原等人。经年累月的辛苦劳作给杨德豫带来了伴随 一生的病痛, 因为肺气肿, 杨德豫住进了医院进行疗 养。1982 年 10 月杨德豫出院, 他做的第一件事就是 着手参与编纂“五四” 以来我国第一套大型外国诗歌 中译本丛书——“诗苑译林”丛书。意味着杨德豫“终 于走上了 “以格律体译格律体”的正确轨道”的第三个 
阶段的代表作一一1981 年出版的《拜伦抒情诗七十首》 就是在这忙碌之余的点滴闲暇中诞生的。

在经历了对于译诗各个阶段的探究之后, 杨德豫 确立了自己“以格律体译格律体”的翻译观，他同市之 琳、孙大雨等人一起, 用“以顿代步”、韵式依照原诗 并且“严宽有度”的方法进行英诗汉译。晚年的杨德豫 性格更加温和、低调、谦逊, 但是依然会在某些涉及 到诗歌翻译的学术问题上与人探讨, 表述自己的观点, 比如他在致商瑞芹的信中与其就“以顿代步”、“韵式 依原诗”等翻译方法据理力争。在坚守着自己的翻译 观的同时, 他抱有一颗审慎和包容之心, 他承认““以 顿代步，韵式依原诗”存在着一定的局限和一些弊病， 也赞同翻译界应该百家争鸣, 自己的流派“仅仅是英 诗汉译众多流派中的一派”。

\section{3.杨译诗歌对原诗格律特征的展现}

在经历了三个阶段、二十余年的摸索之后, 杨德 豫才终于找到了自己的译诗轨道。随着三个阶段的层 层递进, 他的诗歌翻译也逐渐进入到了成熟阶段。以 杨德豫三个阶段的代表作的原诗和译诗为例进行对 比, 笔者发现, 他的译诗在保留原诗的格律特征方面 循序渐进地发生了变化, 使得译诗愈来愈接近原诗的 诗形, 愈来愈能展现出原诗的诗意。如果只翻译出了 字面的意思, 却忽略掉了原诗中的诗学形式, 那么也 不能算得上是一篇翻译的佳作, 因为“诗意就是特定 的意思在特定的诗形的作用下而产生出的一种诗学 效果 (poetic effect) "[8]。由此可见, 诗歌不仅传达“特 定的意思”, 还得在“特定的诗形的作用下”产生诗意。 英诗翻译中也是如此, 无形美, 则不成诗。要想表达 出原诗的诗意, 除了内容之外, 格律特征更加不容忽 视。

杨德豫第一阶段的代表作为 1959 年 10 月第一版 的《朗费罗诗选》。现以其中《普罗米修斯》(Prometheus, or the Poet's Forethought）一诗的第一小节的原诗和译 诗为例来分析原诗和译诗的格律特征:

Of Prometheus, how undaunted

充满了启示和感召,

On Olympus' shining bastions

神话传诵着, 诗歌咏叹着

His audacious foot he planted,

普罗米修斯, 怎样不屈不挠

Myths are told and songs are chanted,

举起他无畏的脚, 踏上

Full of promptings and suggestions.

奥林巴斯山的闪光的稜堡。[9]

原诗的节律为十三节; 行律为每节五行; 音律为 抑扬格; 步律为四音步; 韵律为 $a b a a b$ 。译诗的节律
与行律同原诗一样，但音律、步律和韵律却没有得到 很好的处理, 而后三律才是“格律的核心要素”[10]。

令人感到遗憾的是, 杨德豫在译诗实践中, 他并 没有提及音律的问题，在他的三个阶段的译诗中，对 于原诗音律的翻译一直是缺失的。当代的翻译研究者 已经找到了中国格律诗中与抑扬格相对应的格律元 素——平入。关于平入，杨德豫倒是发表过自己的看 法, 但也仅仅是附带谈一谈, 而且他并没有把平大当 成音律的一部分来讨论，只是说平入安排“值得诗人 和翻译家重视的一种驾驭诗歌语言的艺术和技巧”, 他同意市之琳的说法, 认为平大安排在白话诗中属于 “诗艺”而不属于“诗律”。

这一小节译诗在步律和韵律也毫无规律。关于步 律，这一阶段的杨德豫还没走上“以顿代步”的翻译路 线, 所以暂时还没有将原诗的步律特征表现在译诗当 中, 如上文所说，杨德豫称自己第一阶段的译诗为“半 自由体”，就是因为大多数篇章各行顿数既不整齐也 无规律。韵律也使如此, 虽然第一小节译诗的韵律为 隔行押韵, 韵脚为: 召、挠、堡, 但是其他小节的译 诗并不是如此。以第二小节的译诗为例: 美丽的是那 传说一/说他从天廷的门口逃脱，/是那古的神奇故事 一/说他偷盗和传授了/神仙们的天火。可以看到第二 小节韵律为 aabca, 韵脚为: 说、脱、火, 和第一小节 的隔行押韵的规律是不同的, 而第三小节的韵律和前 两节又都不一样, 就不一一列举了。

可以说，在杨德豫译诗的第一阶段中，除了杨德 豫有意而为的四首诗 (《人生颂》、《少女》、《烈士纪念 日》和《得失》) 在顿数方面整齐有规律地变化以外, 其他的译诗只符合了原诗格律特征中的节律和行律, 忽视了对原诗音律、步律和韵律三方面的翻译，只保 留了原诗很小一部分的诗形, 也就不能较好地展现出 原诗的诗意。

第二阶段的代表作是杨德豫在 1962 年至 1963 年 之间进行翻译，但是延迟到 1978 年出版的《鲁克丽 丝受辱记》 (The Rape of Lucrece), 以其中第一小节 的原诗和译诗为例:

From the besieged Ardea all in post,

Borne by the trustless wings of false desire,

Lust-breathed Tarquin leaves the Roman host,

And to Collatium bears the lightless fire

Which, in pale embers hid, lurks to aspire

And girdle with embracing flames the waist

Of Collatine's fair love, Lucrece the chaste.

淫念熏心的塔昆, 从罗马军营溜号,

离开被围的阿狄亚, 潜赴柯拉廷城堡;

奸猾㔷测的邪欲举双翼将他引导;

他急急忙忙赶路, 揣着无光的火苗一 
这火苗藏在灰灿里，只等时机一到，

要燃起烈焰一团, 前去紧紧环抱

柯拉廷贞淑的妻子——鲁克丽丝的纤腰。[11]

原诗和译诗的节律和行律均一致，而音律一直都 不在杨德豫的考虑范围之内，因此笔者将分析的重点 放在步律和韵律两方面。原诗的步律是五音步，韵律 是 ababbcc。这个时期的杨德豫已经开始有意识地在 用“以顿代步”的方式进行翻译。他认为: “用汉语的顿 （拍、音组）来代替英诗的音步，使译诗每行的顿数 与原诗的音步数相等......在诗行的节奏上获得与原 诗尽可能相似的效果。”因此, 在《鲁克丽丝受辱记》 初译之时, 他也曾试将其译成每行五个顿数, 用顿来 呼应原诗的步, 但是后来发现节奏感不明显, 为了增 强节奏感, 杨德豫在译诗中将每行五个音步变成了 “三三式”一一将五音步英诗改译成每行六顿的汉语 诗, 再用 “半逗律”的方式将六顿拆分为两个三顿。因 此译诗的步律是每行六顿, 三三分开。

在译诗的韵律方面, 杨德豫没有严格地依从原诗, 他原诗将 ababbcc 的韵式改成了每一行都押韵的扁担 韵, 即 aaaaaaa 式, 并且保证全文百分之七十的诗节 都是如此。虽然与原诗的韵律还是相差甚远, 但是从 这一阶段译者对韵律的特殊处理中, 可以看到译者已 经意识到了韵律的问题, 并为此付出努力。杨德豫认 为这样的译诗是 “虽与原诗格律不同但也自有一套谨 严格律的格律诗”。与第一个阶段的译诗比较起来, 第 二阶段的译诗在展现原诗的步律和韵律上进步明显, 杨德豫将这一阶段的译诗称为“准格律体”。由以上分 析的《鲁克丽丝受辱记》的第一小节就可以看出, “准 格律体”译诗体现的不是原诗的格律, 只是译诗本身 有一定的诗学规律, 但也并不是译入语诗歌的传统格 律。相比较“把外国格律诗译成半自由体的白话诗”这 一主张, 它不仅做出了押韵的努力, 在节奏上也有明 显的设计。在节奏方面, 虽然译诗的顿数和原诗的音 步数还是不能做到完全一致, 但是它有意地保持译诗 每行的顿数不变, 而不是像第一阶段那样随意停顿。 综上来看, “准格律体”可以看作是杨德豫的译诗从半 自由体向格律体过渡过程中产生的“半成品”。将第二 阶段的“准格律体”译诗和第三阶段的“以格律体译格 律体”译诗相对比, 可以看到杨译诗歌的第三阶段在 展现原诗的步律和韵律两方面又取得了较大的进展。

杨德豫第三阶段的代表作为 1981 年出版的《拜 伦抒情诗七十首》。以著名的《哀希腊》(The Isles of Greece)第一小节为例:

The Isles of Greece, the Isles of Greece!

希腊/群岛呵, /希腊/群岛!

Where burning Sappho loved and sung, 你有过/萨福/歌唱/爱情,

Where grew the arts of War and Peace, 你有过/隆盛的/武功/文教,
Where Delos rose, and Phoebus sprung! 太阳神/从你的/提洛岛/诞生!

Eternal summer gilds them yet, 长夏的/阳光/还灿烂/如金一

But all, except their Sun, is set. 除了/太阳, /一切/都沉沦! [12]

为了使译诗每行的“顿”更加清楚, 笔者在译诗中 用“”将顿数分开。

这一阶段的杨译诗歌最大的特点就是将“以顿代 步”贯彻到底, 并且韵式依照原诗。原诗的步律为每行 四音步, 译诗为每行四个顿; 原诗的韵律为“ababcc”, 译诗的韵律也是“ababcc”。不同于第二阶段的“准格律 体”译诗, 这一阶段在译诗的韵式和译诗每行的顿数 上明显更加地严格。《拜伦抒情诗七十首》译诗共有 三千四百余行, 绝大多数的译诗的顿数都与原诗的音 步数一致, 韵式也都依照原诗的韵式进行了翻译。至 此, 杨德豫的译诗基本上还原了原诗的步律和韵律, 为展现原诗的节奏美做出了努力, 而节奏恰恰是“诗 歌中最难翻译的诗学成分”, 也是诗歌中“最有价值的 元素”。此后, 杨德豫就一直在“以格律体译格律体”这 条轨道上继续前行, 并以此为翻译规范, 不断地对自 己曾经译过的作品进行修改与校订。

从第一阶段的忽视对原诗的音律、步律、韵律进 行翻译，到第二阶段的尝试去用“顿”来匹配原诗的步 律并重视忠实韵律，再到第三阶段的“以顿代步”和韵 式依原诗, 杨译诗歌在展现原诗的格律特征方面日趋 完善, 愈来愈好地展现了原诗的诗形和诗意。至此, 在诗歌的五个格律特征中, 杨德豫的译诗貌似已经满 足了其中四个，即节律、行律、步律和韵律，但是仍 有问题悬而未决: “以顿代步”是否是能展现原诗的步 律? 原诗的音律应该怎样翻译?

\section{4.对如何在译诗中展现英语格律诗的步律和 音律的反思}

“以顿代步”能展现原诗的步律么? 这其中存在 的最大的问题在于“顿”本身的随意性。杨德豫认为, “顿”, 即自然音组, 以二字顿和三字顿为主, 也可以 有少量的一字顿和四字顿。但是，从格律诗节奏的角 度看, 无论是英诗, 还是汉诗, 节奏都是有规律的、 可预测的，而“顿”在字数上的灵活性却恰恰造成了节 奏的参差不齐。以英诗的五步抑扬格（iambic pentameter) 为例, 每行诗中要包含五个音步, 每个音 步中有两个音节, 而且是以抑扬的声律出现的, 这种 内在的规律才是格律诗的节奏。而译诗中因为“顿”中 字数的不确定性, 就使得译诗长短不一, 和原诗的音 节无法一一对应，因而也失去了原诗的节奏。再者， “顿”的长短不一也很有可能引起读者阅读上、理解上 的不一致，有可能误会“顿”的数目，尤其是一字顿和 四字顿: 一字顿容易让读者误以为它和下一个二字顿 是连在一起; 而四字顿就容易让读者拆分成两个二字 顿。“以顿代步”中的“顿”在字数上的随意性和不确定 
性使得译诗没有比较恰当地保留原诗的步律, 造成了 译诗节奏上的缺失。

为了解决“顿”的字数无规律可循的问题, 王东风 提出了“以逗代步”的诗歌翻译方法, 主张用汉语的 “二字逗”来对应英语格律诗中的占绝大多数的双声 音步, 后又补充了两点: 以一字逗代单声音步, 以三 字逗代三声音步，并且在翻译拜伦的诗歌《哀希腊》 中证明了“以逗代步”此方法的可行性, 具体分析可以 参见王东风所著《以逗代步 找回丢失的节奏》。在“以 逗代步”的翻译诗学观的指引下, 译文不仅能够弥补 “以顿代步”中由于“顿”中字数不确定而产生的杂乱, 更还原了原诗的节奏美, 对英诗翻译的实践有着重大 意义。

再来看英语格律诗中的音律。音律是诗歌中绝对 不能忽视的一个重要组成部分。英语格律诗中的音律 共有六大类: 抑扬格 (iambus)、扬抑格（trochee）、 抑抑扬格 (anapaest)、扬抑抑格 (dactyl)、抑扬抑格 (amphibrach) 和扬扬格 (spondee), 而其中读者比较 常见的, 作者比较常用的就是抑扬格 (iambus)。其实, 英语格律诗主要以抑扬格为主, 其他节奏基本上都被 视为是抑扬格的变体。当然, 在翻译中, 译者对原诗 音律问题的忽视和译者的个人能力是无关的, 因为杨 德豫先生的翻译造诣不需多言, 已经得到了整个翻译 界的认可, 这是和个人的观念以及个人的选择有关的。 如前文所讲, 关于平入的问题, 杨德豫在译文中也会 考虑到, 如有必要, 在译文中他也会尽可能地做出恰 当的安排, 但是在市之琳为核心的“以顿代步”派看来, 平入安排已经超出了基本格律的范畴 (转引自杨德豫, 2012)。因此, 在杨德豫的译文中, 关于原诗的音律, 他并没有做出相应的翻译。

关于如何将英语格律诗中的音律翻译出来这个 问题, 或者说是可否用汉语的平入对应英诗的抑扬这 个问题, 胡适早就说过, 抑扬格就相当于汉语诗的平 入调 ${ }^{[13]}$, 但是包括他自己在内的翻译界学者一直没有 对此进行探索或是比较深入的研究。故此, 是否可以 用汉语的平人对应英诗中的抑扬这个问题就搁置至 今, 逐渐变成了“诗歌翻译研究的一个盲区, 甚至禁 区” ${ }^{[14]}$ 。仍以《希腊群岛》第一小节为例, 原诗的音律 是抑扬格, 每一句都是“抑扬抑扬抑扬抑扬, 由四个抑 扬组成, 节奏整齐并有音乐感。因此, 我们不能无视 的是: 英语诗歌也有音律, 虽然其具体的构成方式与 汉诗的音律不同, 但英诗音律和汉诗音律的生成机制 在很大程度上是一样的: 首先是音差二分式, 英诗是 依音的轻重区分出抑扬, 汉诗是依音的声调区分出平 反; 其次, 是节奏双声式, 英语格律诗的基本节奏是 双音节式, 汉语格律诗也是; 第三, 是节奏变体三声 式, 英语格律诗的节奏变体主要三声式, 汉语格律诗 也是, 五律是上二下三, 七律是二二三。可见, 汉诗 的平入和英诗的抑扬是功能对应的音律结构, 二者的 功能也都一致, 具体功能是建构节奏的音律, 宏观的 功能是诗学功能。因此, 从翻译学的角度看, 二者是 可以互逆的等值体, 理论上讲是可以用来互译的。如
此看来, 平人在针对英语格律诗的翻译时, 非但没有 超出基本格律范畴, 反倒正是其基本的格律范畴了。 对音律翻译的忽视一直都是英诗翻译中的一个弊病。 虽然与汉诗的音律不同，但是英语诗歌音律的存在正 是英诗节奏必不可少的一部分, 值得译者去探索和发 现一种恰当的翻译途径去与之相对应。

从实践的角度看，用汉语的平入去对应英诗的抑 扬, 这样的方法到底可行么? 答案是肯定的。以“平大” 对应“抑扬”并非不可译。早在 2014 年, 王东风就提 出了用平人对应抑扬的翻译诗学观, 并且试译了 The Isles of Greece 的第一节, 结果大获成功, 证明了“以 平人对应抑扬”的可实现性, 也得到了学者们的认同。 “由于平人和抑扬都是交错式双声节奏, 基本特点比 较相似, 这样就决定了它们具有互译的可能性......王 东风的诗歌翻译也恰恰是个很好的实证。”[15]那么, 在诗学功能上, 英语的抑扬和汉语的平入, 是否存在 着翻译对应的理据呢? 这方面的研究在王东风教授 的《以平入代抑扬找回遗落的音美:英诗汉译声律对 策研究》中有详细的分析, 也得出了结论一一汉语的 平人对应英语的抑扬在理论上和实践上是可行的。

如上所说, 从未能更好地展现原诗的步律和音律 方面来看, 杨德豫先生的译诗的确是有缺憾的, 但这 绝非个人的翻译水平问题, 而可以看作是一种时代的 局限, 或者说是一个群体在一个特定的时期内对翻译 中出现的问题的认识不够全面和不够深入而造成的 局限。为了打破这种局限, 王东风教授提出的“以逗代 步”和“以平入代抑扬”的翻译方法, 不仅完善了英诗 的节奏在译诗中的表达, 更开启了英诗汉译的下一阶 段, 即在翻译中展现原诗的节奏性和音乐美。

\section{5.结语}

反观杨德豫先生经历的译诗实践的三个阶段, 在 翻译英语格律诗方面, 他的译诗从没什么规律和约束 可言的“半自由体白话诗”, 到偶尔有一些束缚和制约 的“准格律体诗”，再到成熟时期的“格律体诗”，他的 译诗理念逐渐完善, 译诗质量也逐渐提高。由此可见, 在翻译中, 有些束缚和“脚镣”并不是坏事。没有什么 束缚的“半自由体白话诗”中没有什么节奏可言, 能把 诗歌的意思表达清楚就算是成功, 随着诗歌理念的不 断发展, 诗歌翻译不再只局限于表明含义, 而渐渐地 更倾向于追寻诗歌内在节奏感的“以格律诗译格律诗” 的翻译观。虽然这种译诗理念也并不完善, 但是无数 译者在追求译出格律诗节奏美这条道路上所做出的 努力和付出的心血是值得赞美且令人钦佩的。翻译中 一直被忽视的领域, 如一直被忽视的英诗步律和音律 的翻译问题和译者们意识到却选择视而不见的问题 才是真正的鿭铐, 锁住了翻译发展的前进之路, 也锁 住了译者探索的勇气和进取的心灵。

英诗汉译的下一阶段的发展趋势在于展现原诗 的节奏性和音乐美。杨德豫译诗的三个阶段也折射出 中国近代英诗翻译所经历的几大趋势。五四新诗运动 
以来的百余年之间, 英诗的翻译先后出现了几次大的 翻译趋势, 从最初的把格律诗翻译成自由诗的“自由 化”趋势, 到后来的以顿代步的“顿代化”趋势, 再到现 在更为严谨、更有节奏感的“逗代化”和“平入化”趋势， 舞者身上的“枷锁”越来越多, 难度也随之增大, 但是 译者呈现出的译诗却越来越具有节奏性和音乐美。这 是因为每一代的翻译工作者都在不断地突破前人所 留下的难题, 勇敢地去探索前人未曾涉足的处女地, 也只有如此, 诗歌翻译的领域才能迎来一波接一波的 创新和突破。

\section{REFERENCES:}

[1] Bian, Z.L. (2007). People and poetry. Anhui:Anhui Educational Publishing House.

[2] Jiang, F. (2009). A selection of translation review. Wuhan: Wuhan University Publishing House.

[3] Tu, A.(2010). Tuan's autobiography. Beijing: Sanlian Press.

[4] Bei, T. (2003). On Mr. Yang Deyu's translation of Selected Lyrical Poems of Wordsworth. Chinese Translation.

[5] Yang, D.Y. (2012). Tracing the trail of muse. Hunan:Hunan People's Publishing House.

[6] Fu, Y. (2013). In memory of Yang Deyu. Bookstore.

[7] Yang, D.Y. (2013). Eighty years of my life. Tribute of Social Science.

[8] Wang, D.F. (2018). Poeticity and poetry translating. Foreign Languages Research.

[9] Yang, D.Y. (1959). Selected poems of Longfellow. People's Literature Publishing House.

[10] Wang, D.F. (2014). Retrieving the lost poetic rhythm: A poetic discussion after retranslation of "The Isles of Greece into Chinese. Foreign Language Teaching and Research.

[11] Yang, D.Y. (1978). The rape of Lucrece. People's Literature Publishing House.

[12] Yang, D.Y. (1981). Seventy lyric poems by Byron. Changsha: Hunan People's Publishing House.

[13] Hu,S. (1991). On the writing of sonnets. Hu Shi's comments on poetry. Chengdu: Sichuan Literature and Art Publishing House.

[14] Wang, D.F. (2019). Retrieving the lost musicality:

A study of rhythmic sound pattern in poetry translation. Journal of Foreign Languages.

[15] Zhang, G.K. (2018). "Dou" for foot: A study of Wang Dongfeng's translation poetic. Foreign Languages Resaerch. 